\title{
LIBRI RICEVUTI
}

K. D. Leaver and B. N. Chapman: Thin Films. Wykeham Publications (London) Ltd., London and Winchester, 1971 ; pp. vII-112; $£ 1.50$.

D. C. Montgomery: Theory of the Unmagnetized Plasma. Gordon and Breach, Science Publishers, New York, London, Paris, $1971 ;$ pp. $\mathrm{X}-400 ; \mathfrak{f} 7.30$.

Y. NAKAI, Editor: III International Oonference on Vacuum Ultraviolet Radiation Physics. Tokyo, Japan, Aug. 30-Sept. 2, 1971. The Physical Society of Japan with the financial assiatance of UNESCO, 1971, s.i.p.

(C) by Societa Italiana di Fisica

Proprietà letteraria riservata

Direttore responsabile: GIULIANO TORALDO DI FRANCIA

Stampato in Bologna dalla Tipografla Compositori coi tipi della Tipografla Monograf

Questo fasoicolo è stato licenziato dai torohi il 21-I-1972 